\title{
Identification of a Surface Glutamine Residue (Q64) of Escherichia coli EntA Required for Interaction with EntE
}

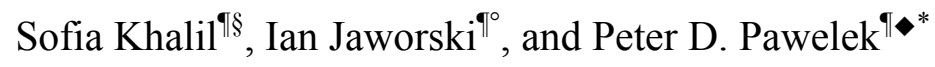 \\ " Department of Chemistry and Biochemistry, Concordia University, 7141 Sherbrooke St., W., \\ Montreal, Quebec, Canada, H4B 1R6 \\ •Groupe de Recherche Axé sur la Structure des Protéines (GRASP)

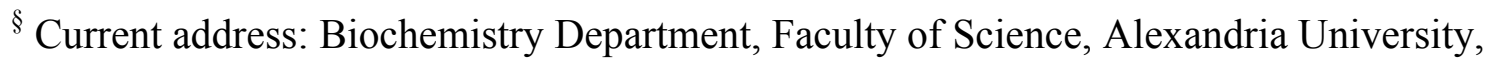 \\ Alexandria, Egypt. \\ ${ }^{\circ}$ Current address: Agilent Technologies, Montreal, Canada. \\ ${ }^{*}$ Correspondence should be addressed to: Peter D. Pawelek, Tel: 514-848-2424 ext. 3118; Fax: \\ 514-848-2868; E-mail: peter.pawelek@concordia.ca.
}

Running title: "Identification of an EntE-binding region on E. coli EntA"

Total number of manuscript words: 4586

Supplementary material: None 


\section{Abstract:}

The enterobactin biosynthetic enzyme EntA forms a complex with EntE, the next enzyme in the pathway, to enhance activation of the enterobactin precursor 2,3-dihydroxybenzoate. Here we used phage display to identify an EntE-interacting region on the surface of EntA. Upon panning immobilized EntE with a random peptide phage library, we recovered 47 unique EntE-binding dodecamer peptide sequences that aligned to a region of the EntA primary sequence corresponding to helix $\alpha 4$. In order to further investigate this region, we mutagenized EntA Q64, a hydrogen-bonding residue found on the surface-exposed face $\alpha 4$. Far-UV circular dichroism, thermal denaturation experiments, and enzymatic assays showed that mutation of EntA residue Gln 64 to alanine (Q64A) had no deleterious effect on EntA structure or function. By following near-UV CD spectral changes, we found that the spectrum of wild-type EntA was altered in the presence of EntE, indicative of conformational changes in EntA aromatic chromophores upon formation of the EntA-EntE complex. However, EntE did not affect the CD spectrum of EntA variant Q64A, demonstrating that this variant did not interact with EntE in a manner similar to wild-type EntA. Analytical ultracentrifugation of wild-type and variant EntA proteins showed that EntA Q64A was predominantly dimeric at $20 \mu \mathrm{M}$, unlike wild-type EntA which was predominantly tetrameric. Taken together, our findings establish that EntA $\alpha 4$ is required for efficient formation of the EntA-EntE as well as for EntA oligomerization.

Keywords: siderophore, enterobactin, protein-protein interactions, phage display, circular dichroism, analytical ultracentrifugation. 
Abbreviations: AUC: analytical ultracentrifugation; CD: circular dichroism; NRPS: nonribosomal peptide synthesis; DHB: 2,3-dihydroxybenzoic acid; Fur: Ferric Uptake Regulator; SDS-PAGE: sodium dodecylsulfate-polyacrylamide gel electrophoresis; TCEP: Tris(2carboxyethyl)phosphine. 


\section{Introduction}

Siderophores are small molecules that chelate ferric iron with extraordinarily high affinity (estimated $K_{D} \sim 10^{-50} \mathrm{M}$ ) [1,2]. Escherichia coli synthesizes and secretes the catecholate siderophore enterobactin for iron acquisition [3-5]. Enterobactin is a cyclic triester of 2,3dihydroxybenzoylserine (DHBS) that is produced in response to low intracellular iron concentrations. Biosynthesis of enterobactin requires the activities of seven cytoplasmic enzymes: EntA-F and EntH. The pathway of enterobactin biosynthesis can be divided into two major arms: (1) the DHB synthetic arm involves the sequential activities of EntC, EntB (Nterminal isochorismatase domain), and EntA to produce 2,3-dihydroxybenzoic acid (DHB) from chorismate; (2) the NRPS arm involves the enzymes EntE, the C-terminal aryl carrier protein domain of holo-EntB (produced by phosphopantetheinylation of apo-EntB by EntD), and EntF. These proteins function together as a 'molecular assembly line' [6] that results in the condensation of three molecules of DHB with three molecules of L-serine followed by cyclization to form enterobactin.

Numerous protein-protein interactions within the NRPS arm have already been reported [7-11]. We recently reported a protein-protein interaction at the functional interface of the DHB synthesis and NRPS arms of the pathway [12]. We discovered that EntA, the last enzyme in the DHB synthesis arm of the pathway, forms a complex with EntE, the enzyme that adenylates DHB for its subsequent attachment to holo-EntB. Formation of the EntA-EntE complex causes an enhancement of EntE activity. EntA produces 2,3-dihydroxybenzoic acid by the NADdependent oxidation of the precursor 2,3-dihydro-2,3-dihydroxybenzoic acid produced by the isochorismatase domain of EntB [13]. The X-ray crystallographic structure of EntA has been reported [14]. EntA has a tetrameric quaternary structure organized as a dimer of dimers (PDB 
code: 2FWM, chains A,B,C,D). One dimer interface (between chains A and C or between chains $\mathrm{B}$ and D) has a buried solvent accessible surface area ( $\triangle \mathrm{ASA})$ of $1,322 \AA^{2}$, whereas the other interface (between chains $\mathrm{A}$ and $\mathrm{B}$ or between chains $\mathrm{C}$ and D) has a buried $\triangle \mathrm{ASA}$ of $995 \AA^{2}$. We have reported that the oligomeric state of EntA in vitro is concentration-dependent: at concentrations below $4 \mu \mathrm{M}$, EntA is predominantly dimeric, whereas at concentrations above 8 $\mu \mathrm{M}$, the protein is predominantly tetrameric [12]. We found that EntE interacts with higher affinity to EntA in the tetrameric form than in the dimeric form.

Here we continue our investigation of EntA-EntE complex formation by identifying an EntA residue required for tetramerization as well as for efficient formation of the EntA-EntE complex. The dual role of this residue suggests that it is located in a region appropriate for targeted disruption of EntA higher-order functions involving protein interactions.

\section{Materials and Methods}

\section{Reagents}

All chemicals were purchased from Bioshop Canada, Inc. (Burlington, Ontario) except 2,3-DHB, which was purchased from Sigma-Aldrich (St. Louis, Mo.) Chorismic acid was kindly provided as a gift by Dr. Joanne Turnbull (Concordia University).

\section{Phage display}

The Ph.D.- $12^{\mathrm{TM}}$ phage display random peptide library kit (New England Biolabs) was used to pan a phage library containing random dodecamer peptide sequences against immobilized EntE. Panning was carried out by incubating $10 \mu \mathrm{l}$ of a library of phage-displayed peptides $\left(2.5 \times 10^{11}\right.$ $\mathrm{pfu} / \mathrm{ml}$ ) in a 24-well polystyrene tray in which each well was coated with approximately $100 \mu \mathrm{g}$ 
of purified EntE. Trays were washed with Buffer A (50 mM Tris (pH 8.6), $150 \mathrm{mM} \mathrm{NaCl}$, and $0.1 \%(\mathrm{v} / \mathrm{v})$ Tween 20$)$, and then blocked with bovine serum albumin ( $1 \mathrm{mg} / \mathrm{ml})$. Bound phage particles were eluted by the addition of a solution containing $0.2 \mathrm{M}$ glycine- $\mathrm{HCl}(\mathrm{pH} 2.2)$ and bovine serum albumin $(1 \mathrm{mg} / \mathrm{ml})$. Eluted phage particles were amplified in E. coli ER2738 cells, and two additional binding/amplification cycles were performed. After three rounds of panning, clones were selected by picking individual plaques containing amplified phage developed on plated lawns of E. coli ER2738 cells. Each plaque represented a clonal phage population containing a single dodecamer peptide sequence. Single-stranded DNA was isolated from phage amplified from plaque picks using the QIAGEN M13 Kit (QIAGEN Inc, Chatsworth, CA) and sequenced at the McGill University and Génome Québec Innovation Centre. Amino acid sequences of dodecamer peptides from isolated phage particles were predicted from DNA sequence data. Bioinformatic analysis of the unique peptide sequences isolated from EntE-bound phage particles was performed using the software RELIC-MATCH [15].

\section{Site-directed mutagenesis}

Site-directed mutagenesis of EntA was performed using the pCA24N-H6-entA construct obtained from the ASKA repository [16] as a template. We previously used this construct for expression and purification of wild-type, recombinant hexahistidine-tagged EntA [12]. Sitedirected mutagenesis experiments were performed using the QuickChange Site-Directed Mutagenesis Kit (Stratagene) along with a pair of custom-synthesized mutagenic oligonucleotide

primers for each mutant generated. Mutagenic oligonucleotides were designed to substitute EntA Gln64 to Ala (Q64A) (forward primer: 5'-GGT CGC GCA AGT GTG TGC GCG ACT GTT AGC TGA AAC G-3'; reverse primer: 5'-CGT TTC AGC TAA CAG TCG CGC ACA CAC 
TTG CGC GAC C-3'). The mutagenesis reaction mixture contained the pCA24N-H6-entA template (5-50 ng), mutagenic forward and reverse primers (125 ng each), $1 \mu \mathrm{l}$ of dNTP mixture (10 mM each of dATP, dTTP, dCTP, and dGTP), and DNA polymerase $(0.05 \mathrm{U} / \mu \mathrm{l})$. The number, duration, and temperature of the PCR cycles were performed according to the manufacturer's instructions. Double-stranded plasmids obtained from site directed mutagenesis were purified using QIAGEN M13 Kit and verified by sequencing.

\section{Expression and purification of EntA wild-type and variant proteins}

Constructs containing DNA sequences encoding in-frame N-terminal hexahistidine tagged EntE, EntA, or EntA variant Q64A were transformed into competent E. coli AG-1 cells (Stratagene). Transformants were grown in LB broth containing $30 \mu \mathrm{g} / \mathrm{ml}$ of chloramphenicol at $37^{\circ} \mathrm{C}$ to an $\mathrm{OD}_{600}$ of 0.8 . Protein expression was induced with the addition of IPTG to $1 \mathrm{mM}$ followed by further incubation at $37^{\circ} \mathrm{C}$ for 3 hours. Cell lysis was performed using the BugBuster Master Mix lysis buffer (Novagen). Clarified lysates were applied to a $10 \mathrm{ml}$ Profinity IMAC column connected to a BioLogic DuoFlow FPLC system (Bio-Rad Laboratories). The column was washed using Buffer B (50 mM Hepes (pH 8.0), $100 \mathrm{mM} \mathrm{KCl,} 1 \mathrm{mM}$ TCEP, and $10 \mathrm{mM}$ imidazole). The hexahistidine-tagged proteins were eluted using a linear gradient of imidazole $(10 \mathrm{mM}$ to $500 \mathrm{mM})$ in Buffer B. Fractions containing the highest $\mathrm{OD}_{280}$ fractions were analyzed by SDS-PAGE, pooled, and dialyzed against Buffer C (50 mM Hepes (pH 8.0), $100 \mathrm{mM} \mathrm{KCl,} 1$ $\mathrm{mM}$ TCEP, and $15 \%$ glycerol) followed by storage at $-20^{\circ} \mathrm{C}$. For coupled enzyme assays, Nterminal hexahistidine-tagged E. coli EntC and EntB were overexpressed and purified similar to the approach described above. 


\section{Circular dichroism spectroscopy}

Far-UV CD spectra of purified EntA and EntA variant proteins were collected on a Jasco J-815 circular dichroism spectropolarimeter over the range $200-260 \mathrm{~nm}$ in $0.2-\mathrm{cm}$ path-length rectangular cell. Proteins were dialysed into Buffer D (Tris ( $\mathrm{pH} 8.0$, adjusted using $\mathrm{H}_{2} \mathrm{SO}_{4}$ ), 0.5 $\mathrm{mM}$ TCEP, $100 \mathrm{mM} \mathrm{NaCl}$, and $15 \%$ glycerol) and then diluted to a concentration of $5 \mu \mathrm{M}$ in the same buffer. Spectra were recorded at $20^{\circ} \mathrm{C}$ by averaging five wavelength scans $(1 \mathrm{~nm}$ bandwidth) in $0.2-\mathrm{nm}$ steps at a rate of $100 \mathrm{~nm} / \mathrm{min}$, and $0.25 \mathrm{sec}$ response. For thermal stability experiments, ellipticities at $222 \mathrm{~nm}$ were monitored between $20{ }^{\circ} \mathrm{C}$ and $65{ }^{\circ} \mathrm{C}$ (rate of temperature change $=0.25{ }^{\circ} \mathrm{C} \mathrm{min}^{-1}$ ) using a Jasco $\mathrm{J}-815 \mathrm{CD}$ spectropolarimeter with a temperature-controlled cell holder. Thermal denaturation data were used to determine the fraction of protein folded at each temperature point by assigning the ellipticity values at $222 \mathrm{~nm}$ collected at $20^{\circ} \mathrm{C}$ as $100 \%$ folded protein (fraction folded $=1.0$ ), and assigning the $222 \mathrm{~nm}$ ellipticity values collected at $65{ }^{\circ} \mathrm{C}$ as $100 \%$ unfolded protein (fraction folded $=0.0$ ). Near-UV CD spectra of EntA wild-type or EntA Q64A variant proteins (20 $\mu \mathrm{M})$, EntE (20 $\mu \mathrm{M})$, and equimolar mixtures of EntE with wild-type EntA or EntA variant were collected on a Jasco J-710 CD spectropolarimeter over the range $250-320 \mathrm{~nm}$ using a $1 \mathrm{~cm}$ path-length rectangular cell. To obtain spectra of EntA wild-type or Q64A variant in the presence of EntE, the EntE near-UV CD spectrum was subtracted from those of corresponding mixture spectra. All spectra were recorded at $20^{\circ} \mathrm{C}$ by averaging three wavelength scans ( $1 \mathrm{~nm}$ bandwidth) in $0.2-\mathrm{nm}$ steps at a scan speed of $20 \mathrm{~nm} / \mathrm{min}$, with a 2 -second response time. All spectra of protein solutions were corrected for contributions of buffer components in the absence of protein. 


\section{Coupled enzyme activity assay}

Activities of purified wild-type and variant EntA Q64A proteins were assayed in the presence of purified EntB and EntC, which were used to enzymatically convert chorismate to the EntA substrate 2,3-dihydro-2,3-dihydroxybenzoate. EntA activity was measured spectrophotometrically by following the reduction of $\mathrm{NAD}^{+}$to $\mathrm{NADH}$ at $340 \mathrm{~nm}$. Reaction

mixtures (total volume $1 \mathrm{~mL}$ ) contained $1 \mu \mathrm{M}$ EntA, $10 \mu \mathrm{M}$ EntC, $10 \mu \mathrm{M}$ EntB, $50 \mathrm{mM}$ Tris (pH 7.5), $50 \mathrm{mM} \mathrm{NaCl}, 10 \mathrm{mM} \mathrm{MgCl}_{2}$, and $300 \mu \mathrm{M}$ chorismate. For steady-state kinetics assays, $\mathrm{NAD}^{+}$concentrations were varied between $1 \mathrm{mM}$ and $5 \mathrm{mM}$. Reactions were initiated by adding chorismate to the reaction mixture pre-equilibrated at $37{ }^{\circ} \mathrm{C}$. Activities were measured at $37{ }^{\circ} \mathrm{C}$ as the linear increase in $[\mathrm{NADH}]$ over a time-period of one minute. NADH concentrations were calculated using the molar extinction coefficient for NADH at $340 \mathrm{~nm}\left(6,220 \mathrm{M}^{-1} \mathrm{~cm}^{-1}\right)$. All assays were performed in triplicate, and the data were fit to the Michaelis-Menten steady-state kinetics model using Kaleidagraph 4.5 (Synergy Software).

\section{Analytical ultracentrifugation}

Sedimentation velocity experiments were performed in a Beckman XL-I analytical ultracentrifuge with a four-place An-60Ti rotor. Wild-type EntA and EntA Q64A variant proteins were dialyzed into Buffer D (50 mM Tris buffer $(\mathrm{pH} 8.0), 150 \mathrm{mM} \mathrm{NaCl}$ and $0.5 \mathrm{mM}$ TCEP) and adjusted to final concentrations of $2 \mu \mathrm{M}$ or $20 \mu \mathrm{M}$ by dilution with Buffer D. Samples were centrifuged at $40,000 \mathrm{rpm}$ for approximately $10 \mathrm{~h}$ at $20^{\circ} \mathrm{C}$; for each run a total of 250 absorbance scans were collected in which optical density at $280 \mathrm{~nm}$ was measured. Data were analyzed by fitting to the $g\left(s^{*}\right)$ distribution using the computer program DCDT+ (v2.2.1) [17]. Approximately 20 scans were selected from each run of 250 scans for analysis. 


\section{Results and Discussion}

\section{Identification of an EntE-Interacting Region on the Surface of EntA}

We employed phage display to identify residues occurring at the EntA-EntE protein interaction interface. The Ph.D.- $12^{\mathrm{TM}}$ phage display random peptide library was used to pan against pure, immobilized EntE to obtain a set of unique EntE-binding peptide sequences. After three successive rounds of panning we obtained 47 unique dodecamer peptide sequences isolated from phage particles that specifically bound to EntE (Table 1). These peptides were used as input for the RELIC server program RELIC-MATCH [15]. Bioinformatic analysis of the peptide sequences using RELIC-MATCH identified a subset of the 47-peptide set that aligned as a cluster to EntA primary amino acid sequence between residues 60-71 (Fig. 1A). Such a clustering of peptides indicates a high probability that the corresponding region of the EntA primary sequence is found at the EntA-EntE interaction interface. Examination of the EntA crystal structure (PDB code: 2FWM) revealed that these residues occur on the EntA surface, at the exterior face of EntA helix $\alpha 4$ (Fig. 1B, red shading). Helix $\alpha 4$ consists of four turns and on chain B is within approximately $10 \AA$ of the chain D $\alpha 5$ (D- $\alpha 5)$ at the B-D dimerization interface (Fig. 1C). The A-chain $\alpha 4$ helix is similarly positioned relative to $\mathrm{C}-\alpha 5$ at the A-C interface. Within the boundaries of the cluster defined by our phage display results, the residues oriented towards the solvent-exposed surface are found on three turns of $\alpha 4$ : A60, Q64, A68 (Fig. 1A, asterisks). At the center of this region is Q64, a bulky hydrogen bond donor. Immediately adjacent to Q64 is the charged residue R65; this residue is unlikely to participate in the EntA-EntE interaction interface since the EntA 
crystal structure indicates that the R65 side-chain is oriented away from the solvent-exposed face of EntA a4, such that it forms an intrachain ionic interaction with EntA E49.

\section{Expression, purification and structural characterization of EntA Q64A}

Guided by our phage display outcomes, we used site-directed mutagenesis to generate a Glu-toAla mutation at EntA position 64 in order to determine the role of Q64 in EntA-EntE complex formation and EntA oligomerization. We overexpressed EntA Q64A variant protein with purified the variant protein to near $(\sim 90 \%)$ homogeneity. SDS-PAGE analysis of purified wildtype and variant EntA proteins indicated that Q64A had a subunit molecular mass similar to wild-type EntA ( $28 \mathrm{kDa}$ ) (data not shown). We performed biophysical experiments to test the structural integrity of purified recombinant Q64A variant protein. Far-UV CD spectra showed that wild-type EntA in solution has major ellipticity minima at $208 \mathrm{~nm}$ and $222 \mathrm{~nm}$ (Fig. 2A, solid line) consistent with the significant helical content in this protein as revealed by its X-ray crystallographic structure (PDB code: 2FWM). The far-UV CD spectrum of Q64A (Fig. 2A, dashed line) was found to be nearly superimposable with wild-type EntA, indicating no significant change in secondary structure. We further tested the EntA Q64A variant by measuring changes in ellipticity at $222 \mathrm{~nm}$ as a function of increasing temperature. The thermal denaturation curve of EntA Q64A was observed to be similar to that of wild-type EntA (Fig. 2B, dashed line), indicating that the overall fold of Q64A was similar to that of wild-type EntA (Fig. 2B, solid line). 


\section{The Q64A variant is not enzymatically impaired}

We employed a coupled enzyme assay to determine if the enzymatic activity of the Q64A variant was comparable to wild-type EntA. Michaelis-Menten kinetic analysis revealed that the Q64A variant had similar apparent $\mathrm{K}_{\mathrm{m}}$ and $\mathrm{k}_{\text {cat }}$ values (denoted $\mathrm{K}_{\mathrm{m}}$ ' and $\mathrm{k}_{\text {cat }}$ ', respectively) compared to wild-type EntA (Table 2). Both enzymes exhibited $\mathrm{K}_{\mathrm{m}}{ }^{\prime}$ values for $\mathrm{NAD}^{+}$in the low millimolar range, and had similar apparent turnover numbers. In terms of overall catalytic efficiency $\left(\mathrm{k}_{\mathrm{cat}}{ }^{\prime} / \mathrm{K}_{\mathrm{m}}{ }^{\prime}\right)$, wild-type and Q64A variant are within a two-fold difference of each other. The retention of catalytic activity by EntA Q64A is consistent with the comparable thermal melting behavior that we observed for both the wild-type and variant proteins, demonstrating similar overall folding of the monomer subunits.

\section{EntA residue Gln64 is required for efficient interaction with EntE}

To experimentally verify our phage display outcomes, we investigated the effect of the Q64A mutation on EntA-EntE complex formation. We employed a near-UV CD spectroscopic perturbation assay that we previously used to investigate the EntE-EntB interaction [8]. Near-UV circular dichroism spectra were collected for wild-type EntA and the Q64A variant protein following mixture with an equimolar concentration of purified EntE. A near-UV CD spectrum of pure EntE was also collected, and EntE spectral contributions were then subtracted from the protein mixture spectra. Comparison of the subtracted spectra with those of EntA or Q64A in the absence of EntE revealed conformational alterations of aromatic chromophores (Phe, Trp, Tyr) occurring upon protein complex formation. Using this approach, we observed ellipticity increases in the near-UV spectrum (250-320 nm) of wild-type EntA upon mixture with EntE (Fig. 3A), corresponding to perturbations in the conformations of EntA aromatic chromophore 
residues due to the presence of EntE. The EntE-perturbed spectrum of wild-type EntA showed maximal signal differences around $270 \mathrm{~nm}$ and $290 \mathrm{~nm}$, corresponding to perturbations in the environments of Phe and Trp residues [18]. The spectral changes observed for wild-type EntA may be due to either direct perturbation of surface chromophores, and/or to indirect conformational rearrangements occurring upon protein complexation. It is notable that EntA $\alpha 5$ has three aromatic residues sharing the same surface as Gln 64: Trp 96, Phe 100, Phe 108 (Fig. 1B, green-shaded surface). In contrast to the wild-type enzyme, we did not observe an alteration in the near-UV spectrum of the EntA variant Q64A in the presence of EntE, indicating no EntEinduced conformational changes (Fig. 3B). The insensitivity of the Q64A variant to the presence of EntE demonstrates that EntA residue Gln 64 has a direct involvement in formation of the EntA-EntE complex in vitro. Although it is not possible from this assay to determine if the Q64A mutation completely abolishes the EntA-EntE interaction, our data clearly show that residue 64 is necessary for efficient EntA-EntE complex formation.

\section{EntA residue Gln64 is required for tetramerization}

Given the proximity of EntA $\alpha 4$ to the B-D dimer interface, we were interested in investigating oligomerization behavior of the EntA Q64A variant. We performed sedimentation velocity AUC experiments on wild-type EntA and the Q64A variant at low $(2 \mu \mathrm{M})$ and high $(20 \mu \mathrm{M})$ concentrations at which wild-type EntA was predicted to be found as dimers and tetramers, respectively. At a protein concentration of $2 \mu \mathrm{M}$ (Fig. 4A), $\mathrm{g}\left(\mathrm{s}^{*}\right)$ profiles of our sedimentation velocity data show that both wild-type EntA and Q64A occur predominantly as dimers. At a protein concentration of $20 \mu \mathrm{M}$, wild-type EntA (Fig. 4B, black circles) occurs predominantly as tetramer, consistent with our previously reported observations, whereas the Q64A variant (Fig. 
4B, grey squares) sediments at a value intermediate between dimer and tetramer, indicating that the equilibrium position for this variant has been shifted towards a dimeric species. The EntA Xray crystallographic structure reveals that Gln64 participates in a hydrogen bond network (Fig. 1C) involving $\alpha 4$ and $\alpha 5$ of one chain (B or A) and $\alpha 5$ of the interacting chain (D or C, respectively) in the dimer. Within this network, Gln64 forms a hydrogen bond with Gln113 on the same chain, thus orienting B- $\alpha 5$ to interact with D- $\alpha 5$ via hydrogen bonds and weak electrostatic interactions. The Q64A mutation would affect positioning of B- $\alpha 5$ relative to D- $\alpha 5$, thus disrupting the 4-helix bundle required for formation of the higher-affinity dimers (B-D, A$\mathrm{C} ; 1,322 \mathrm{~A}^{2}$ ). Such a disruption would result in reduced tetramerization with corresponding enrichment of the weaker-affinity dimers (A-B, C-D; $995 \mathrm{~A}^{2}$ ), consistent with our AUC observation. Although removal of the Gln side chain at position 64 would be consistent with disruption of the higher-affinity (and thus likely the more physiologically relevant) dimer, our outcomes reported here strongly support our previously reported observation that optimal EntAEntE interaction requires EntA in the tetrameric form, and we now have identified a key residue required for both EntA tetramerization and EntA-EntE interaction.

\section{Author Contributions}

S.K. performed phage display experiments, site-directed mutagenesis, protein expression and purification, near-UV circular dichroism experiments, AUC experiments and data analysis, preparation of draft manuscript, and manuscript revision; I.J. performed protein expression and purification, enzyme kinetics assays, far-UV circular dichroism experiments, thermal melting experiments, and manuscript revision; P.D.P. performed manuscript revision and communication, and assisted with AUC experiments and data analysis. 


\section{Acknowledgements}

This work was supported by Discovery Grant 341983 from the Natural Sciences and Engineering Research Council of Canada to PDP. The authors thank Daniel Foshag and Michael Lowden for helpful discussions regarding this manuscript. They also acknowledge the Concordia BIOFINS platform for access to its CD spectropolarimeters, and the Concordia Centre for Structural and Functional Genomics for access to its analytical ultracentrifuge. Sofia Khalil was supported by a scholarship from the Cultural Affairs and Missions Sector, Egypt. 


\section{References}

1. Harris W.R., Carrano C.J., Cooper S.R., et al. (1979) Coordination chemistry of microbial iron transport compounds. 19. Stability constants and electrochemical behavior of ferric enterobactin and model complexes. J. Am. Chem. Soc. 101: 6097-6104.

2. Miethke M., Marahiel M.A. (2007) Siderophore-based iron acquisition and pathogen control. Microbiol. Mol. Biol. Rev. 71: 413-451.

3. de Lorenzo V., Bindereif A., Paw B.H., Neilands J.B. (1986) Aerobactin biosynthesis and transport genes of plasmid ColV-K30 in Escherichia coli K-12. J. Bacteriol. 165: 570-578.

4. Raymond K.N., Dertz E.A., Kim S.S. (2003) Enterobactin: an archetype for microbial iron transport. Proc. Natl. Acad. Sci. USA 100: 3584-3588.

5. Valdebenito M., Crumbliss A.L., Winkelmann G., Hantke K. (2006) Environmental factors influence the production of enterobactin, salmochelin, aerobactin, and yersiniabactin in Escherichia coli strain Nissle 1917. Int. J. Med. Microbiol. 296: 513-520.

6. Crosa J.H., Walsh C.T. (2002) Genetics and assembly line enzymology of siderophore biosynthesis in bacteria. Microbiol. Mol. Biol. Rev. 66: 223-249.

7. Lambalot R.H., Gehring A.M., Flugel R.S., et al. (1996) A new enzyme superfamily - the phosphopantetheinyl transferases. Chem. Biol. 3: 923-936.

8. Khalil S., Pawelek P.D. (2009) Ligand-Induced Conformational Rearrangements Promote Interaction between the Escherichia coli Enterobactin Biosynthetic Proteins EntE and EntB. $J$. Mol. Biol. 393: 658-671.

9. Drake E.J., Nicolai D.A., Gulick A.M. (2006) Structure of the EntB multidomain nonribosomal peptide synthetase and functional analysis of its interaction with the EntE adenylation domain. Chem. Biol. 13: 409-419. 
10. Lai J.R., Fischbach M.A., Liu D.R., Walsh C.T. (2006) Localized Protein Interaction Surfaces on the EntB Carrier Protein Revealed by Combinatorial Mutagenesis and Selection. $J$. Am. Chem. Soc. 128: 11002-11003.

11. Lai J.R., Fischbach M.A., Liu D.R., Walsh C.T. (2006) A protein interaction surface in nonribosomal peptide synthesis mapped by combinatorial mutagenesis and selection. Proc. Natl. Acad. Sci. USA 103: 5314-5319.

12. Khalil S., Pawelek P.D. (2011) Enzymatic adenylation of 2,3-dihydroxybenzoate is enhanced by a protein-protein interaction between Escherichia coli 2,3-dihydro-2,3-dihydroxybenzoate dehydrogenase (EntA) and 2,3-dihydroxybenzoate-AMP ligase (EntE). Biochemistry 50: 533545.

13. Liu J., Duncan K., Walsh C.T. (1989) Nucleotide sequence of a cluster of Escherichia coli enterobactin biosynthesis genes: identification of entA and purification of its product 2,3dihydro-2,3-dihydroxybenzoate dehydrogenase. J. Bacteriol. 171: 791-798.

14. Sundlov J.A., Garringer J.A., Carney J.M., et al. (2006) Determination of the crystal structure of EntA, a 2,3-dihydro-2,3-dihydroxybenzoic acid dehydrogenase from Escherichia coli. Acta Crystallogr. D Biol. Crystallogr. 62: 734-740.

15. Mandava S., Makowski L., Devarapalli S., Uzubell J., Rodi D.J. (2004) RELIC - a bioinformatics server for combinatorial peptide analysis and identification of protein-ligand interaction sites. Proteomics 4: 1439-1460.

16 Kitagawa M., Ara T., Arifuzzaman M., et al. (2005) Complete set of ORF clones of Escherichia coli ASKA library (a complete set of E. coli $\mathrm{K}-12$ ORF archive): unique resources for biological research. DNA Res. 12: 291-299. 
17. Philo J.S. (2006) Improved methods for fitting sedimentation coefficient distributions derived by time-derivative techniques. Anal. Biochem. 354: 238-246.

18 Kelly S.M., Jess T.J., Price N.C. (2005) How to study proteins by circular dichroism. Biochim. Biophys. Acta - Proteins and Proteomics 1751: 119-139. 


\begin{tabular}{|c|c|}
\hline \multicolumn{2}{|c|}{$\begin{array}{l}\text { Table 1. EntE-binding peptides obtained by } \\
\text { phage display. }\end{array}$} \\
\hline ADANRHSTLRER & TPLWQHLLSGRA \\
\hline ADANRRSTLRER & TPPWVTVLLSRQ \\
\hline ADSRRALLAQRA & TPQWQQLLSFRQ \\
\hline ADSWRALLAQRA & TPSWATLLAQRA \\
\hline ASPWHQLLAERR & TYRMDIMSIKTV \\
\hline ATSWKEMLAERQ & WHDITSLRQYSF \\
\hline DSPPIFDATLPK & TPWEEVLLSRLR \\
\hline DWREILGARSQV & DPVWVNILTSRQ \\
\hline EHVLWQQLLTSR & AQWQETLSERAR \\
\hline ENWRLTLLQRNG & KCCYYDHSHALS \\
\hline GGLHWTEILRSR & HSLRSDWPLRPG \\
\hline GGVHWSEILSYR & ELTGWRLLLAQR \\
\hline GRLQQHEIFRSG & KVVDLYSGWNRS \\
\hline KTPWQEMLASRI & HVLWQHVVDLCR \\
\hline PPMWADMLLARS & GPFLPLTSLHWR \\
\hline SCKQVLEHRQGM & NWRETMGVRSQV \\
\hline SIDGRS I ISSRN & SDWTHVLSQRAL \\
\hline SLDWTELLRLRT & KIYDLSLLHPST \\
\hline SMPQWQELLKVR & QPAWQQTLINRS \\
\hline SPLWQDI ILTRS & SWMETLRTRNMS \\
\hline SPTYHSSTGLND & SLSNYQIAGNGL \\
\hline SSPSWRDVLLSR & TVSWQALLEMRQ \\
\hline STGDWREILRNR & TPAWQATLLGRQ \\
\hline TDWRTQLHLRQG & \\
\hline
\end{tabular}


Table 2. Wild-type and variant EntA proteins: steady-state enzyme kinetics. ${ }^{a}$

\begin{tabular}{|c|c|c|c|}
\hline Protein & $\begin{array}{l}K_{m}^{\prime} b \\
(\mathrm{uM})\end{array}$ & $\begin{array}{c}k_{c a t}{ }^{\prime} \\
\left(\mathrm{min}^{-1}\right)\end{array}$ & $\begin{array}{c}k_{c a t}{ }^{\prime} / K_{m}^{\prime} \\
\left(\min ^{-1} \mathrm{uM}^{-1}\right) \\
\times 10^{2}\end{array}$ \\
\hline wild-type EntA & $2370+740$ & $73.5+10.1$ & 3.1 \\
\hline Q64A & $1960 \pm 540$ & $39.5 \pm 4.4$ & 2.0 \\
\hline \multicolumn{4}{|c|}{$\begin{array}{l}{ }^{a} \text { Apparent kinetic parameters determined from coupled enzyme } \\
\text { assay (see Methods). Data were fit to the Michaelis-Menten } \\
\text { steady-state kinetic model. Mean values }(n=3) \text { of kinetic } \\
\text { parameters are reported, along with standard errors of the fit. }\end{array}$} \\
\hline
\end{tabular}




\section{Figure Legends}

\section{Figure 1: Identification of EntE-interacting residues on EntA.}

(A) RELIC-MATCH output showing alignment of phage-derived EntE-binding dodecapeptide sequences to the primary sequence of E. coli EntA. Residues shaded in black indicate positions of sequence identity. Residues shaded in grey indicate positions of sequence similarity. Asterisks indicate residues on helix $\alpha 4$ with similar side-chain orientations. The boundaries of helix $\alpha 4$ are indicated by a solid line. (B) X-ray crystallographic structure of the EntA monomer subunit (PDB code: 2FWM) shown as a cartoon with a semi-transparent molecular surface. The EntEinteracting surface identified by phage display corresponding to $\alpha 4$ is colored red. The surface of the adjacent $\alpha 5$ helix is colored green. Aromatic chromophore residues on EntA $\alpha 5$ sharing the same surface as Q64 and A68 are shaded in green. Figure was generated using Pymol.

(C) Cartoon representation of EntA tetramer structure (PDB code: $2 \mathrm{FWM}$ ) zoomed in on the interface between $\mathrm{B}$ and $\mathrm{D}$ chains. Secondary structure elements and carbon atoms corresponding to EntA tertiary structure subunits are colored as follows: B-chain = cyan; Dchain $=$ yellow. The coordinates of the side-chain oxygen and nitrogen atoms on B-Q113 were swapped relative to the PDB coordinates to reflect $180^{\circ}$ rotation around $\mathrm{C} \gamma-\mathrm{C} \delta$. Dotted lines with numbers represent interatomic distances in Ångstrom units. Figure was generated using Pymol.

Figure 2: Far-UV circular dichroism of EntA wild-type and variant proteins.

(A) Far-UV spectra of EntA wild-type and variant proteins collected at $20{ }^{\circ} \mathrm{C}$. EntA wild-type (solid line), and Q64A (dashed line). (B) Thermal denaturation of EntA wild-type and variant 
proteins. Relative ellipticities measured at $222 \mathrm{~nm}$ are represented as fraction folded over temperature range shown: EntA wild-type (solid line), Q64A (dashed line).

\section{Figure 3: Near-UV spectra of EntA wild-type and variant proteins in the presence or absence of EntE.}

Near-UV CD spectra were collected at $20{ }^{\circ} \mathrm{C}$ for each EntA protein sample (wild-type or variant) and then compared to the spectrum of that protein when mixed with an equimolar concentration of EntE after subtraction of the contribution of EntE to the spectrum. (A) wild-type EntA (black circles), wild-type EntA in the presence of EntE (white circles); (B) Q64A (black circles), Q64A in the presence of EntE (white circles).

Figure 4. Sedimentation velocity analytical ultracentrifugation of wild-type EntA and Q64A variant.

Purified protein samples were centrifuged at $40,000 \mathrm{rpm}\left(\right.$ temp $=20{ }^{\circ} \mathrm{C}$ ) in a Beckman XL-I analytical ultracentrifuge. Sedimentation boundaries were analyzed using DCDT+ and shown here as $\mathrm{g}\left(\mathrm{s}^{*}\right)$ plots. (A) Wild-type EntA (black circles) and Q64A variant (grey squares) at low concentration (2 $\mu \mathrm{M})$; (B) Wild-type EntA (black circles) and Q64A variant (grey squares) at high concentration $(20 \mu \mathrm{M})$; 
Figure 1.

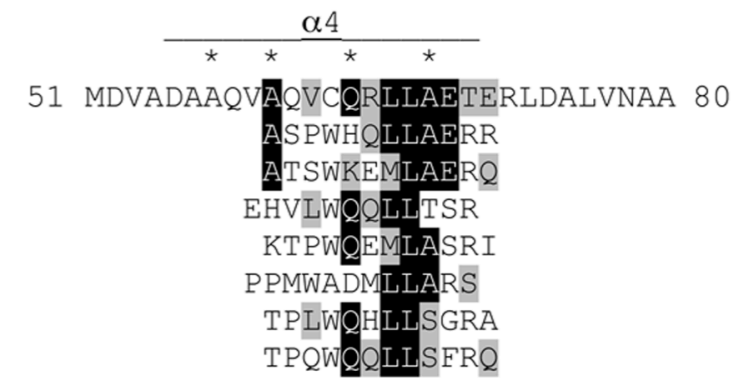

B
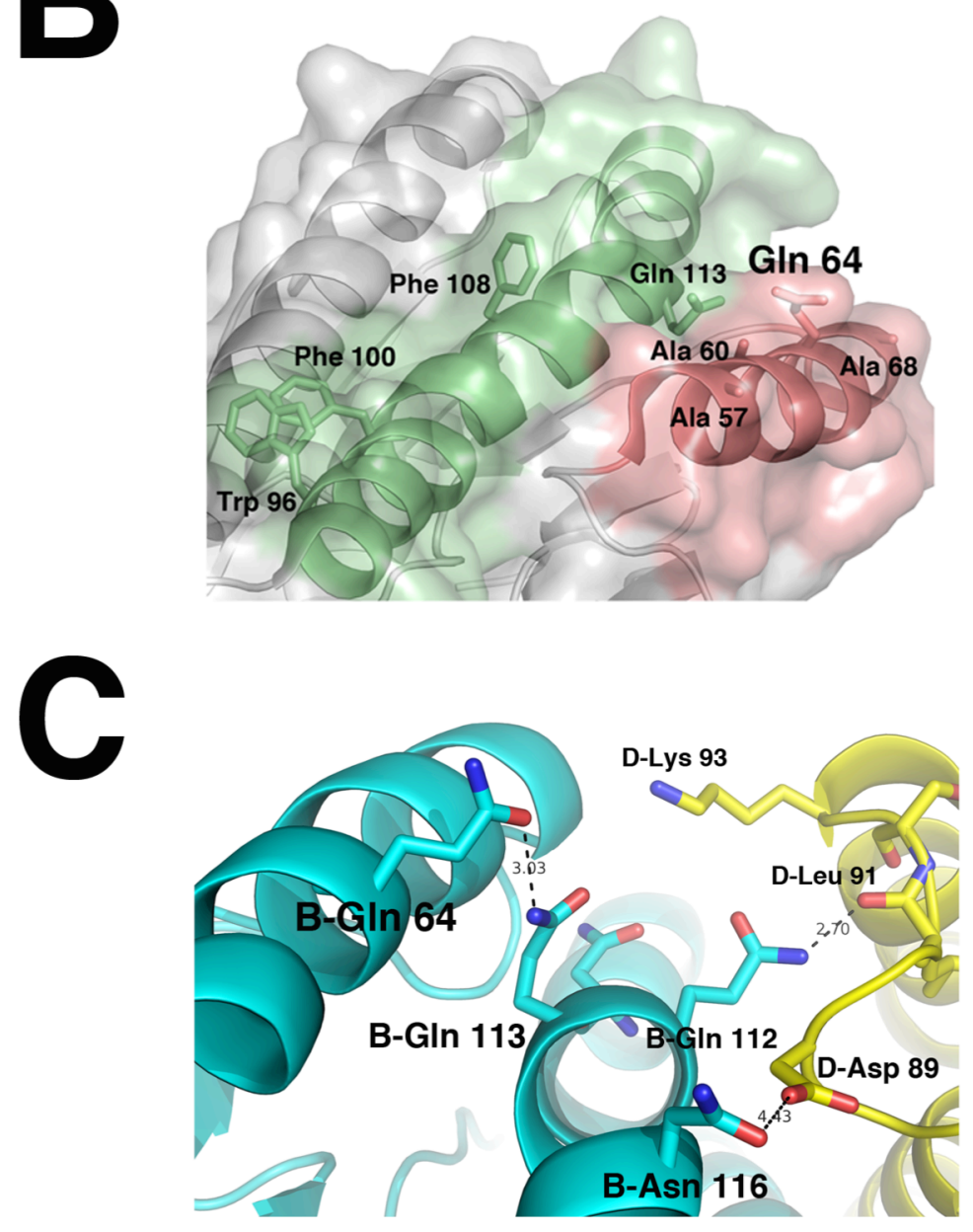
Figure 2.

A

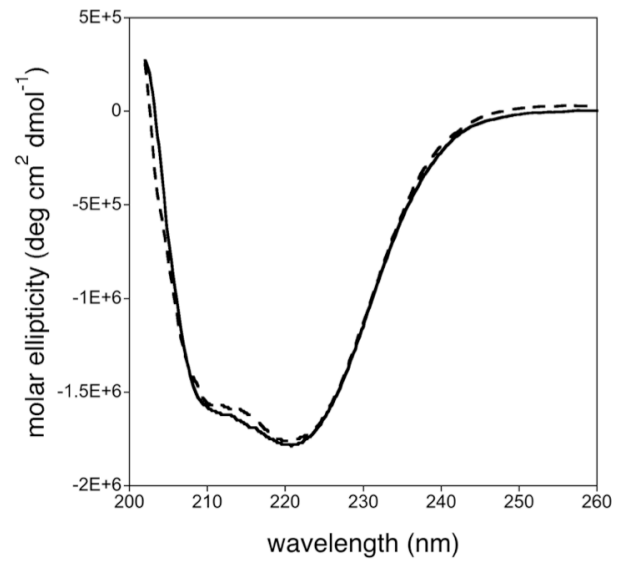

B

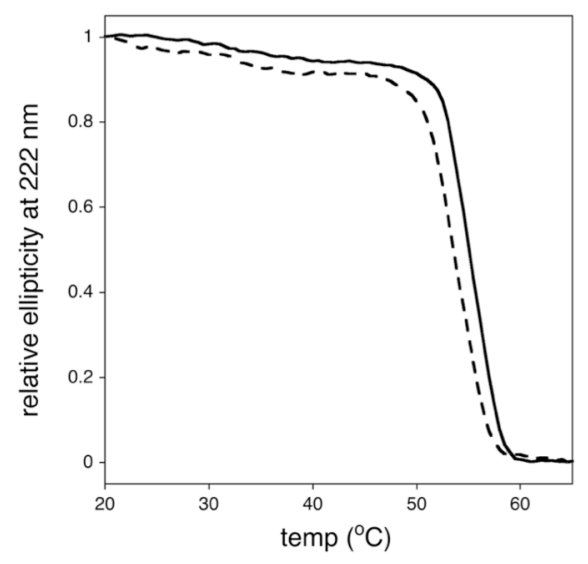


Figure 3.
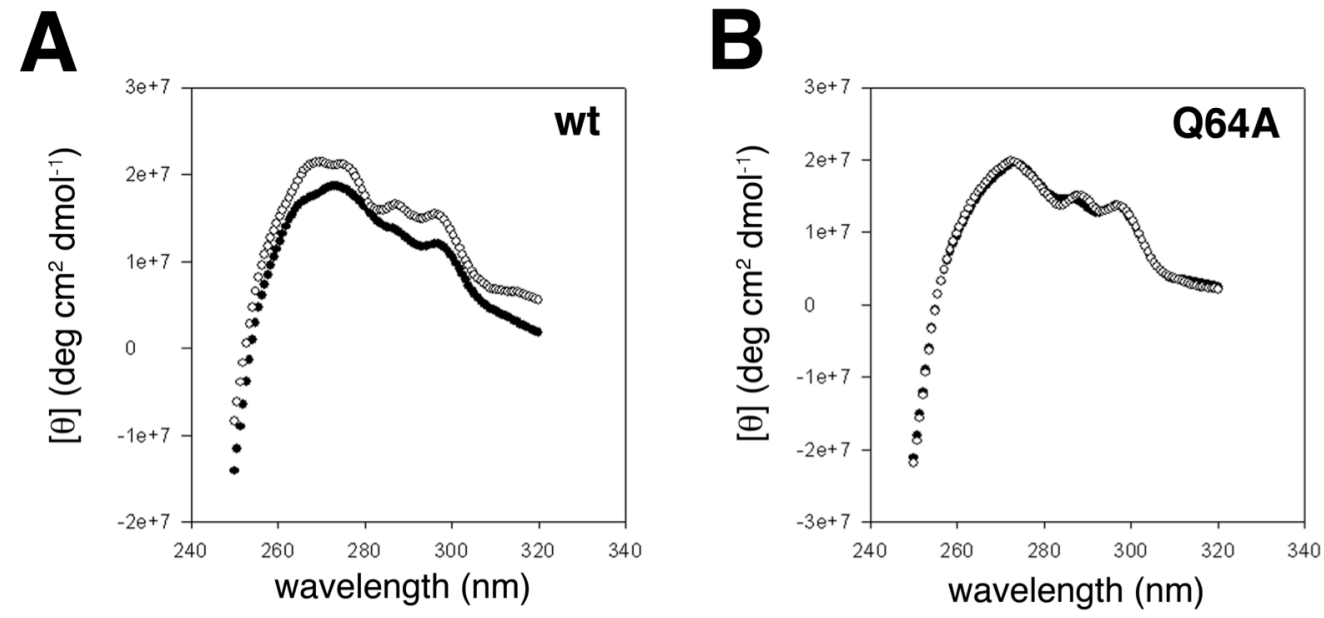
Figure 4.

A

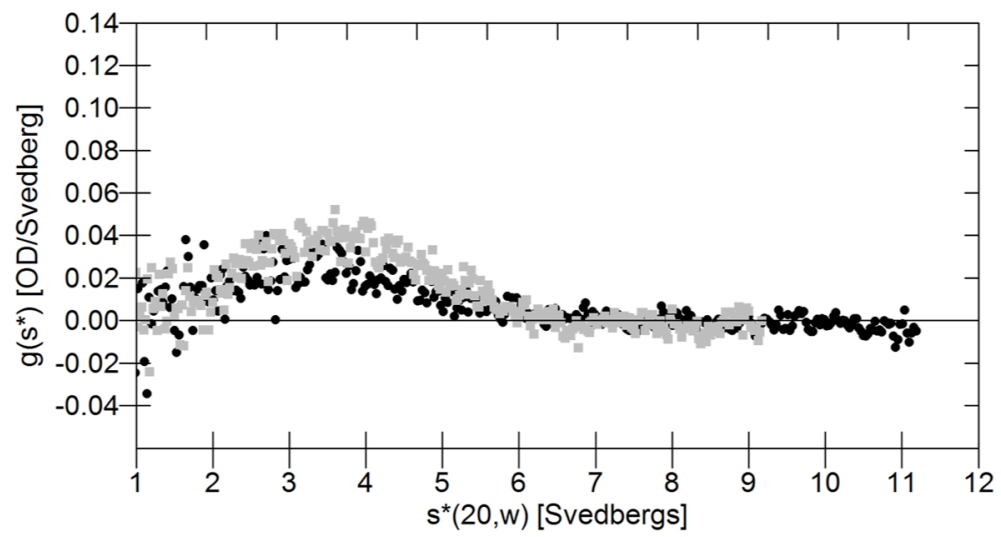

B

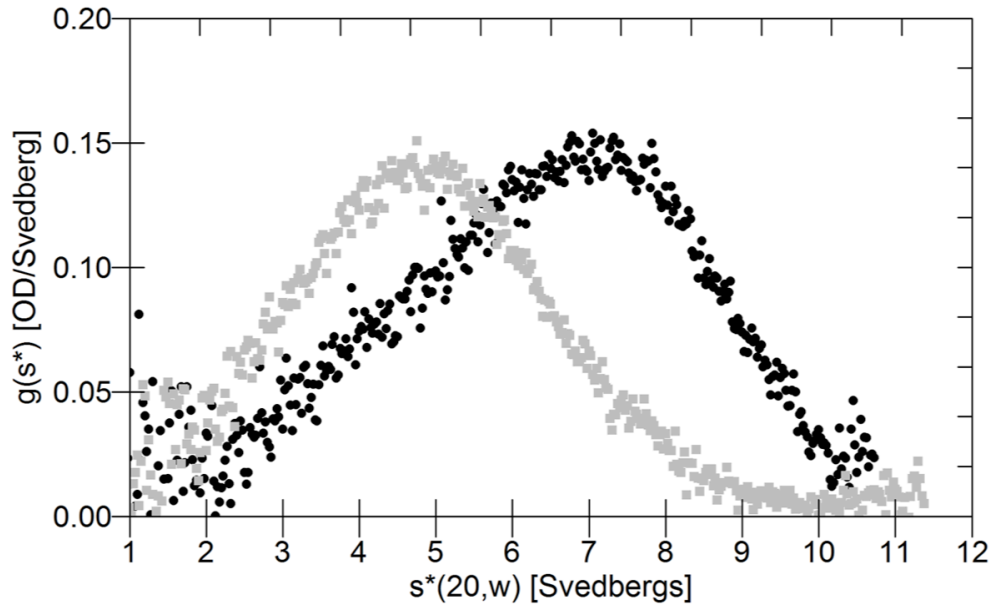

